\title{
Nuclear densities and the statistics of nucleonic constituents
}

\author{
M. Betz \\ Instituto de Física, Universidade Federal do Rio Grande do Sul, Caixa Postal 15051, 91501-970 Porto Alegre, \\ Rio Grande do Sul, Brazil \\ D. Hadjimichef \\ Instituto de Física Teórica, Universidade Estadual Paulista, Rua Pamplona, 145, 01405-900 São Paulo, \\ São Paulo, Brazil
}

(Received 20 January 1994)

\begin{abstract}
In the quark model of the nucleon, the Fermi statistics of the elementary constituents can influence significantly the properties of multinucleon bound systems. In the Skyrme model, on the other hand, the basic quanta are bosons, so that qualitatively different statistics effects can be expected a priori. In order to illustrate this point, we construct schematic one-dimensional quark and soliton models which yield fermionic nucleons with identical baryon densities. We then compare the baryon densities of a two-nucleon bound state in both models. Whereas in the quark model the Pauli principle for quarks leads to a depletion of the density in the central region of the nucleus, the soliton model predicts a slight increase of the density in that region, due to the bosonic statistics of the meson-field quanta.
\end{abstract}

PACS number(s): 21.10.Gv, 12.39.-x, 12.60.Rc, 11.10.Lm

\section{INTRODUCTION}

Quantum chromodynamics (QCD) is generally accepted as the fundamental theory of strong interactions [1]. In QCD, hadrons should emerge as composite systems of elementary quarks and gluons. However, because of the difficulty in solving QCD in the nonperturbative regime, hadronic structure has so far mainly been approximated by semiphenomenological models that incorporate some essential properties of QCD, such as confinement and chiral symmetry.

The implications of nucleon structure for nuclear physics have been intensely investigated in recent times [2]. On the one hand, as our knowledge of the former increases, the models developed to describe the latter may need to be improved or revised. Conversely, one may hope that the large body of knowledge accumulated in nuclear physics could be used to distinguish between possible pictures of nucleon structure. In view of the rather large uncertainties in the details of the dynamics of nucleon models, it seems desirable to concentrate the attention on their main qualitative features, such as the statistics of the building blocks.

Probably the simplest and most traditional model of hadron structure is the nonrelativistic quark model, in which the nucleon is described, in first approximation, as a system of three independent quarks confined in a central potential [3]. The Fermi statistics of quarks belonging to different nucleons has been shown to have significant implications in nuclear structure [4], being responsible, for example, for a sizable contribution to the so-called European Muon Collaboration (EMC) effect.

An alternative to quark models is afforded by soliton models of the Skyrme [6] type, in which nucleons appear as topologically nontrivial configurations in a nonlinear meson field theory. The possible relation of these models to QCD, as argued by 't Hooft [7] and Witten [8], has led to their revival during the past decade [9]. Simple questions related to statistics are challenging in such models since, even though the elementary quanta are bosons, the soliton must possess an overall fermionic character. Anyway, effects associated with the bosonic statistics of the constituents would be expected to arise, when nucleons overlap in a nuclear bound state.

It is the purpose of the present work to explore some of the implications of quantum statistics in a bound state of topological solitons, performing a comparison with a bound state of quark clusters. In view of the difficulties mentioned in the preceding paragraph, we shall restrict ourselves to the study of schematic "toy" models in one spatial dimension. Only in this framework are we able to construct a creation operator for a fermionic extended soliton - an essential tool for studying a multisoliton bound state. The soliton model is defined such as to produce the same baryon density, for a single nucleon, as a simplified quark model. We then calculate and compare the baryon densities of a two-nucleon bound state in both models.

It must be emphasized that the one- and two-nucleon states used to calculate the baryon densities are not derived from specific dynamical models. Rather they are constructed in such a way as to possess the essential features which would be expected to emerge, mutatis mutandis, from similar realistic models in three spatial dimensions.

This paper is organized as follows. In Sec. II we define a schematic quark model and derive the expression of the baryon density distribution of a two-nucleon bound state in this model. In Sec. III we construct a creation operator for a one-dimensional fermionic soliton, with topological charge density equal to the baryon density of the quark model of Sec. II. We set up the formalism necessary to 
calculate the baryon density distribution of the two-body bound state in this model also. In Sec. IV we present numerical results obtained in both models. We display quantum statistics effects by comparing the results with those obtained by convolution of the baryon density for a single nucleon with the probability density for the relative position of the nucleons in the bound state. Section V contains our conclusions and some speculations.

\section{SCHEMATIC QUARK MODEL}

The influence of quark statistics on the properties of a nuclear bound state has already been studied by several authors [4]. Here we shall consider a schematic model in one-dimensional space, without spin or flavor. The idealized nucleon is described as a cluster of three independent quarks carrying different colors. The operator creating such a nucleon centered at $y$ is

$\Psi^{\dagger}(y)=\int \prod_{i=1}^{3} d x_{i} \varphi\left(x_{i}-y\right) q_{r}^{\dagger}\left(x_{1}\right) q_{b}^{\dagger}\left(x_{2}\right) q_{g}^{\dagger}\left(x_{3}\right)$

where $q_{\alpha}^{\dagger}(x)$ creates at $x$ a quark of color $\alpha(\alpha=r, b, g)$ and $\varphi(x)$ is the normalized single-quark wave function.

Although in this case there would be no difficulty in working with a more realistic model, we wish to treat both kinds of model (quark and soliton) at the same level of sophistication in order to facilitate comparison. In a realistic three-dimensional soliton model, the construction of anticommuting creation operators for nucleons still represents an unsurmounted task, although attempts can be found in the literature [5]. In the same spirit, center-of-mass motion has not been removed in (1), since this operation would be awkward in the soliton model.

The quark creation and destruction operators satisfy standard anticommutation relations:

$$
\left\{q_{\alpha}(x), q_{\alpha^{\prime}}^{\dagger}\left(x^{\prime}\right)\right\}=\delta_{\alpha, \alpha^{\prime}} \delta\left(x-x^{\prime}\right) .
$$

The quark wave function will be assumed to be Gaussian:

$$
\varphi(x)=\frac{1}{\left(\sqrt{2 \pi} x_{0}\right)^{\frac{1}{2}}} \exp \left(-\frac{x^{2}}{4 x_{0}^{2}}\right),
$$

where $x_{0}$ is the rms size of the nucleon.

The baryon density of a nucleon centered at the origin is

$$
\rho_{N}(x)=\left\langle 0\left|\Psi(0) \hat{\rho}(x) \Psi^{\dagger}(0)\right| 0\right\rangle,
$$

where $|0\rangle$ denotes the vacuum state and the baryon density operator is given by

$$
\hat{\rho}(x)=\frac{1}{3} \sum_{\alpha} q_{\alpha}^{\dagger}(x) q_{\alpha}(x)
$$

From (1) and (2) one obtains immediately

$$
\rho_{N}(x)=|\varphi(x)|^{2}=\frac{1}{\sqrt{2 \pi} x_{0}} \exp \left(-\frac{x^{2}}{2 x_{0}^{2}}\right) .
$$

The (unnormalized) state of two nucleons centered at $y$ and $-y$, respectively, is

$$
|y\rangle=\Psi^{\dagger}(y) \Psi^{\dagger}(-y)|0\rangle
$$

A bound state of two composite nucleons can be written as a linear superposition of such states:

$$
|D\rangle=\frac{1}{\sqrt{N}} \int d y \zeta(y)|y\rangle
$$

where $\zeta(y)$ is a wave function describing the relative motion of the clusters. Since the nucleons are fermions and do not carry spin or isospin, this wave function must be antisymmetric. We shall choose the form

$$
\zeta(y)=2\left(\frac{3 \sqrt{6}}{\sqrt{\pi} d^{3}}\right)^{\frac{1}{2}} y \exp \left(-3 y^{2} / d^{2}\right),
$$

where $d$ is the rms value of $2 y$, the distance between the cluster centers. The normalization constant appearing in (8) is given by

$$
N=\int d y d y^{\prime} \zeta^{*}\left(y^{\prime}\right) \zeta(y)\left\langle y^{\prime} \mid y\right\rangle .
$$

The overlap between states of two localized clusters can be calculated using (7), (1), and (2). One obtains

$$
\begin{aligned}
\left\langle y^{\prime} \mid y\right\rangle= & \Delta^{6}\left(y^{\prime}-y\right)-3 \Delta^{4}\left(y^{\prime}-y\right) \Delta^{2}\left(y^{\prime} \dashv y\right) \\
& +3 \Delta^{2}\left(y^{\prime}-y\right) \Delta^{4}\left(y^{\prime}+y\right)-\Delta^{6}\left(y^{\prime}+y\right),
\end{aligned}
$$

with

$$
\Delta(u)=\int d x \varphi^{*}(x) \varphi(x+u)=\exp \left(-\frac{u^{2}}{8 x_{0}^{2}}\right) .
$$

The baryon density of the "deuteron" is given by

$$
\begin{aligned}
\rho_{D}(x) & =\langle D|\hat{\rho}(x)| D\rangle \\
& =\frac{1}{N} \int d y d y^{\prime} \zeta^{*}\left(y^{\prime}\right) \zeta(y)\left\langle y^{\prime}|\hat{\rho}(x)| y\right\rangle .
\end{aligned}
$$

The matrix elements of the density operator between states of two localized nucleons are easily worked out using (7), (1), (5), and (2). One gets

$$
\left\langle y^{\prime}|\hat{\rho}(x)| y\right\rangle=\sum_{\nu, \nu^{\prime}= \pm} \nu^{\prime} \nu \mathcal{R}\left(x ; \nu^{\prime} y^{\prime}, \nu y\right)
$$

where

$$
\begin{aligned}
\mathcal{R}\left(x ; y^{\prime}, y\right)= & \varphi^{*}\left(x-y^{\prime}\right) \varphi(x-y) \Delta\left(y^{\prime}-y\right) \\
& \times\left[\Delta^{4}\left(y^{\prime}-y\right)-2 \Delta^{2}\left(y^{\prime}-y\right) \Delta^{2}\left(y^{\prime}+y\right)\right. \\
& \left.+\Delta^{4}\left(y^{\prime}+y\right)\right] .
\end{aligned}
$$

The last two terms in the square brackets in the above expression are due to quark exchange between the nucleons. Using (9)-(15), the baryon density of the "deuteron" and the constant $N$ can be obtained in closed form. The final expressions are somewhat complicated and will not be displayed here. 


\section{TOPOLOGICAL SOLITON MODEL}

As already mentioned, a fermionic creation operator for a topological soliton in a realistic three-dimensional model, such as the Skyrme model, is not available. On the other hand, in one-dimensional space, a soliton creation operator has been constructed by Mandelstam [10]. This operator creates a pointlike soliton of unit topological charge in a field theory possessing an $S_{1}$ topology [11]. In such a theory, the topological charge density is proportional to the space derivative of the field $\phi(x)$ :

$$
\hat{\rho}(x)=\frac{1}{2 \pi} \phi^{\prime}(x) .
$$

We shall generalize the Mandelstam construction in order to obtain an operator which creates an extended soliton state in which the topological charge density is equal to the baryon density (6) of the quark model discussed in the preceding section. We use the ansatz [12]

$$
\Psi^{\dagger}(y)=\lambda \exp \left[A^{\dagger}(y)\right]
$$

with

$$
\begin{aligned}
A^{\dagger}(y)= & 2 \pi i \int d \xi f(y-\xi) \pi(\xi) \\
& +\frac{i}{2} \int d \xi g(\xi-y) \phi(\xi)
\end{aligned}
$$

Here $\pi(x)$ is the canonical conjugate of $\phi(x), \lambda$ is a normalization constant, and $f$ and $g$ are complex functions to be determined. The Mandelstam operator is regained by substituting for $f$ the Heaviside $\theta$ function and for $g$ the Dirac $\delta$ function.

A first condition to be imposed on this operator is that it should carry unit topological charge, which requires

$$
\left[\int d x \hat{\rho}(x), \Psi^{\dagger}(y)\right]=\Psi^{\dagger}(y)
$$

With the help of the canonical commutation relations of the field operators, one easily derives from (17) and (18) that

$$
\left[\int d x \hat{\rho}(x), \Psi^{\dagger}(y)\right]=[f(\infty)-f(-\infty)] \Psi^{\dagger}(y)
$$

Condition (19) can therefore be fulfilled by imposing

$$
f(-\infty)=0, f(\infty)=1
$$

so that $f$ possesses the same asymptotic behavior as the $\theta$ function which appears in the original Mandelstam operator.

A further constraint on the functions $f$ and $g$ follows from the requirement that the solitons obey Fermi statistics, i.e.,

$$
\left\{\Psi^{\dagger}(x), \Psi^{\dagger}(y)\right\}=0 .
$$

From (17) and (18), we derive

$$
\left\{\Psi^{\dagger}(x), \Psi^{\dagger}(y)\right\}=\left\{1+\exp \left[A^{\dagger}(x), A^{\dagger}(y)\right]\right\} \Psi^{\dagger}(y) \Psi^{\dagger}(x)
$$

A straightforward calculation of the commutator appearing in this expression shows that (22) will be satisfied if

$$
\int d \xi f(x-\xi) g(\xi-y)=\theta(x-y)
$$

This condition was also satisfied by the original Mandelstam operator.

The last condition, which will allow the complete determination of the functions $f$ and $g$, is that the topological charge density corresponding to a soliton centered at the origin be given by (6). This density must be calculated according to (4), using the soliton creation operator (17), the density operator (16), and the usual Fock vacuum of the scalar field theory. From (16), it is clear that this condition specifies, up to an integration constant, the expectation value of the field in the one-soliton state. Since the field manifold is assumed to possess an $S_{1}$ topology, this mean field must tend to a multiple of $2 \pi$ at large distance. A possible choice of the integration constant yields the condition

$$
\begin{aligned}
\langle 0| \Psi(0) \phi(x) & \Psi^{\dagger}(0)|0\rangle \\
& =-2 \pi+\frac{\sqrt{2 \pi}}{x_{0}} \int_{-\infty}^{x} d x \exp \left(-\frac{x^{2}}{2 x_{0}^{2}}\right) .
\end{aligned}
$$

In order to implement this last condition, as well as (24), it is convenient to expand the field and its conjugate momentum in a plane wave basis, in standard fashion, and rewrite (17) and (18) in the form

$$
\Psi^{\dagger}(y)=\mu \exp \left[A_{+}^{\dagger}(y)\right] \exp \left[A_{-}^{\dagger}(y)\right]
$$

where $\mu$ is a redefined normalization constant and

$$
\begin{aligned}
& A_{+}^{\dagger}(y)=\int d k e^{-i k y} \sqrt{\frac{\omega_{k}}{2}} \sigma(k) a^{\dagger}(k), \\
& A_{-}^{\dagger}(y)=\int d k e^{i k y} \sqrt{\frac{\omega_{k}}{2}} \chi(k) a(k) .
\end{aligned}
$$

Here $a^{\dagger}(k)$ and $a(k)$ are creation and annihilation operators for the bosonic quanta and $\omega_{k}=\sqrt{k^{2}+m^{2}}$, with $m$ the mass of these quanta. The functions $\sigma$ and $\chi$ are combinations of the Fourier transforms of $f$ and $g$,

$$
\begin{aligned}
& \sigma(k)=-2 \pi f(-k)+\frac{i}{2 \omega_{k}} g(k), \\
& \chi(k)=2 \pi f(k)+\frac{i}{2 \omega_{k}} g(-k) .
\end{aligned}
$$

The constant $\mu$ is fixed by requiring that the one-soliton state, obtained by applying the creation operator onto the Fock vacuum, be normalized. This gives

$$
\mu^{-2}=\exp \left[C_{1}(0)\right]
$$

with 


$$
\begin{aligned}
C_{1}\left(y-y^{\prime}\right) & \doteq\left[A_{+}\left(y^{\prime}\right), A_{+}^{\dagger}(y)\right] \\
& =\frac{1}{2} \int d k e^{i k\left(y-y^{\prime}\right)} \omega_{k}|\sigma(k)|^{2} .
\end{aligned}
$$

The Fourier transform of (24) reads

$$
f(k) g(k)=\frac{1}{2 \pi(i k+\eta)},
$$

where $\eta$ is a small positive quantity which specifies how the pole at $k=0$ is to be handled. From (26)-(28) it follows that

$$
\left\langle 0\left|\Psi(0) \phi(x) \Psi^{\dagger}(0)\right| 0\right\rangle=\frac{1}{\sqrt{2 \pi}} \int d k e^{i k x} \sigma(k) .
$$

Using this result and condition (25), the function $\sigma$ is easily determined:

$$
\sigma(k)=\frac{\sqrt{2 \pi}}{i k-\eta} \exp \left(-\frac{x_{0}^{2} k^{2}}{2}\right) .
$$

Finally, inverting (29) and (30), inserting the resulting expressions in (33) and solving for $\chi$, we obtain

$$
\begin{aligned}
\chi(k)= & \frac{1}{\sqrt{2 \pi} \omega_{k}(i k+\eta)} \\
& \times\left[-k+\sqrt{k^{2}+4 \pi^{2} \omega_{k}^{2} e^{-2 x_{0}^{2} k^{2}}}\right] e^{x_{0}^{2} k^{2} / 2} .
\end{aligned}
$$

We observe that, from $(29),(30),(35)$, and (36), it follows that

$$
f(k \rightarrow 0) \simeq \frac{1}{\sqrt{2 \pi}(i k+\eta)},
$$

which is the Fourier transform of the $\theta$ function. Therefore the large distance behavior of $f$ is compatible with (21). In fact, there exists another solution for the function $\chi$, differing from (36) by the sign in front of the square root, but it leads to a function $f$ which does not satisfy (21) and must therefore be rejected.

The construction of a creation operator satisfying the required conditions is thus completed. However, the mass $m$ of the field quantum is undetermined. In the Skyrme model, this would be the pion mass. In view of this, noting that the pion Compton wavelength is about $\sqrt{2}$ times the nucleon size, we have set $m=\left(\sqrt{2} x_{0}\right)^{-1}$ in the calculations discussed in the next section.

The calculation of the baryon density in the two-soliton bound state is based on (13), (10), and (9), with the operators and states of the soliton model now substituting their quark model correspondents. The matrix elements of the density operator (16) between states of two localized solitons are easily evaluated by passing the creation and destruction operators for the field quanta through the soliton operators. One obtains

$$
\begin{aligned}
\left\langle y^{\prime}|\hat{\rho}(x)| y\right\rangle= & \frac{1}{2}\left[\rho_{N}\left(x-y^{\prime}\right)+\rho_{N}\left(x+y^{\prime}\right)\right. \\
& \left.+\rho_{N}(x-y)+\rho_{N}(x+y)\right]\left\langle y^{\prime} \mid y\right\rangle .
\end{aligned}
$$

A similar procedure yields the overlap between states of two localized solitons:

$$
\begin{aligned}
\left\langle y^{\prime} \mid y\right\rangle= & \exp \left\{C_{2}(-2 y)+C_{2}^{*}\left(-2 y^{\prime}\right)\right. \\
& \left.+2\left[C_{1}\left(y+y^{\prime}\right)+C_{1}\left(y-y^{\prime}\right)-C_{1}(0)\right]\right\},
\end{aligned}
$$

with $C_{1}$ given by (32) and

$$
\begin{aligned}
C_{2}\left(y-y^{\prime}\right) & \doteq\left[A_{-}^{\dagger}\left(y^{\prime}\right), A_{+}^{\dagger}(y)\right] \\
& =\frac{1}{2} \int d k e^{-i k\left(y-y^{\prime}\right)} \omega_{k} \chi(k) \sigma(k) .
\end{aligned}
$$

Thus the calculation of the overlap (39) requires the evaluation of the Fourier transforms (32) and (40). The procedure followed to handle the singularities present in the functions $\sigma$ and $\chi$ is sketched in the Appendix. Once the overlap (39) is cast in a form suitable for computation, the baryon density of the two-body bound state can be obtained by evaluating numerically the integrals (13) and (10).

\section{NUMERICAL RESULTS}

Since in the quark model the baryon density of the two-body bound state depends only on the parameters $x_{0}$ and $d$, it can be seen easily to possess the following scaling property:

$\rho_{D}\left(x ; x_{0}, d\right)=\frac{1}{x_{0}} \rho_{D}\left(\frac{x}{x_{0}} ; 1, \frac{d}{x_{0}}\right) \doteq \frac{1}{x_{0}} \tilde{\rho}_{D}\left(\frac{x}{x_{0}} ; \frac{d}{x_{0}}\right)$,

where the dependence in the parameters has been displayed and the last equality defines the adimensional baryon density $\tilde{\rho}_{D}$. In fact, once the choice $m=$ $\left(\sqrt{2} x_{0}\right)^{-1}$ is made, this is also true in the soliton model. Therefore, it is sufficient to study the function $\tilde{\rho}_{D}$ for various values of the ratio $d / x_{0}$ of bound-state size to nucleon size.

In order to evaluate the importance of quantum statistics effects, it is useful to compare the baryon density derived from the quantum-mechanical bound states to that obtained by a simple convolution of the density for a single nucleon with the probability distribution of the nucleon centers in the bound state, i.e.,

$$
\begin{aligned}
\rho_{D}^{(c)}\left(x ; x_{0}, d\right) & =2 \int d y|\zeta(y)|^{2} \rho_{N}(x-y) \\
& \doteq \frac{1}{x_{0}} \tilde{\rho}_{D}^{(c)}\left(\frac{x}{x_{0}} ; \frac{d}{x_{0}}\right),
\end{aligned}
$$

where the scaling property has been used again to define the adimensional density $\tilde{\rho}_{D}^{(\mathrm{c})}$.

Numerical results for a few values of $d / x_{0}$ are shown in Fig. 1. It is seen that the Pauli principle for quarks leads to a strong depletion of the density in the central region of the bound state. In contrast, for all but the smallest value of $d / x_{0}$, a small increase is observed in the soliton model. This effect, which was expected in view of the 

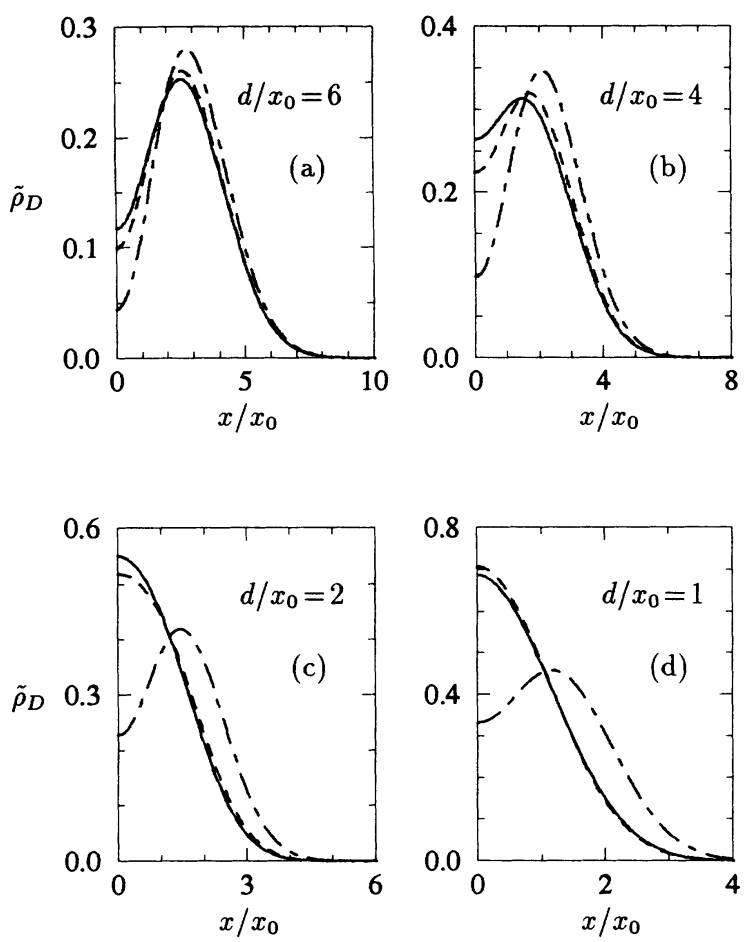

FIG. 1. The adimensional baryon density of the two-nucleon bound state for various values of the ratio $d / x_{0}$ of bound-state size to nucleon size. Full line, soliton model; dot-dashed line, quark model; dashed line, convolution of the single-nucleon density with the probability density for the relative position of the nucleon centers.

Bose statistics of the field quanta, is perhaps surprisingly small. We note, however, that in a state of two localized solitons, the baryon density would be [see (38)]

$$
\frac{\langle y|\hat{\rho}(x)| y\rangle}{\langle y \mid y\rangle}=\rho_{N}(x-y)+\rho_{N}(x+y) .
$$

This is simply the superposition of the densities of individual nucleons, without quantum overlap effects. On the other hand, in the quark model, it can easily be seen from (14) and (15) that quantum statistics effects are present for localized nucleons also. The peculiar result (43) holds because of two circumstances: (i) the baryon density (16) is linear in the field and (ii) the state of two localized solitons is a coherent state [13] in which the expectation value of any function of the field operator is the corresponding classical function of the mean field.

\section{CONCLUSIONS}

The influence of the statistics of nucleonic constituents on the baryon density of a two-nucleon bound state has been studied in schematic one-dimensional models.

In the quark model, the Pauli principle is responsible for a significant suppression of the density in the central region of the bound state. In the soliton model, on the other hand, the Bose statistics of the basic quanta results in only a small increase of the density in that region.

The smallness of the effect obtained in the soliton model can be at least partly attributed to the fact that the baryon (topological) density is a linear function of the field operator. This feature is peculiar to one-dimensional topological soliton models, so that the conclusion that statistics effects are small may not persist in a more realistic model. In any case, in three as well as in one dimensions, a soliton is described quantum mechanically by a coherent state, which is a superposition of many quanta in different linear momentum states. This can be contrasted with the quark model, in which only a small number of different states are relevant. On this basis, one would expect statistics effects to be more significant in quark models. Of course, our schematic model overestimates these effects since it disregards spin and isospin.

Since our purpose was to compare statistics effects, we have constructed our models in such a way as to yield identical baryon densities for a single nucleon. In fact, the baryon number distribution of the nucleon in the Skyrme model possesses a hole at the center, whereas the standard quark model yields a maximum. In addition, we have not considered dynamical effects, which could also influence bound-state densities.

The mesons making up the topological soliton have been considered here as structureless elementary quanta. The fact that these mesons are of course themselves quark-antiquark bound states implies further statistics effects, which can safely be neglected only if the mesons are sufficiently small compared to the nucleon.

The analysis of baryon densities presented here could of course be applied to any charge density of interest, as well as to other nuclear properties. Calculations in realistic models would be needed to assess the possibility of exploiting such quantum statistics effects to distinguish between models of the nucleon experimentally.

\section{ACKNOWLEDGMENTS}

We are grateful to G. Krein and Th. A. J. Maris for arousing our interest in the issue of constituent statistics in nuclear physics. This work was supported by the following Brazilian agencies: Financiadora de Estudos e Projetos (FINEP), Fundação de Amparo à Pesquisa do Estado do Rio Grande do Sul (FAPERGS), Fundação de Amparo à Pesquisa do Estado de São Paulo (FAPESP), and Conselho Nacional de Desenvolvimento Científico e Tecnológico (CNPq).

\section{APPENDIX}

In order to cast the overlap between localized twosoliton states in a form suitable for numerical evaluation, we first consider the functions $C_{1}$ and $C_{2}$ defined by (32) and (40), respectively. Using (35), we get

$$
C_{1}(u)=2 \pi \int_{0}^{\infty} d k \cos k u \frac{\omega_{k}}{k^{2}+\eta^{2}} e^{-x_{0}^{2} k^{2}}
$$


Separating the contribution from the pole of the integrand at $k=0$, we rewrite this expression in the form

$$
C_{1}(u)=\frac{\pi^{2} m}{\eta} e^{-\eta|u|}+\tilde{C}_{1}(u)
$$

with

$$
\tilde{C}_{1}(u)=2 \pi \int_{0}^{\infty} d k \cos k u\left(\omega_{k} e^{-x_{0}^{2} k^{2}}-m\right) / k^{2} .
$$

Similarly, from (35) and (36), we get

$$
\begin{aligned}
C_{2}(u)= & -i \int_{0}^{\infty} d k \sin k u \frac{k}{k^{2}+\eta^{2}} \\
& -\int_{0}^{\infty} d k \cos k u \frac{\sqrt{k^{2}+4 \pi^{2} \omega_{k}^{2} e^{-2 x_{0}^{2} k^{2}}}}{k^{2}+\eta^{2}} .
\end{aligned}
$$

The first term of this expression can be written immediately in terms of the Heaviside step function. In the second term, the contribution from the pole of the integrand at $k=0$ can again be separated. It is also necessary to isolate the logarithmic singularity of this term at $u=0$. Performing these manipulations, we derive

$$
\begin{aligned}
C_{2}(u)= & -i \frac{\pi}{2}[\theta(u)-\theta(-u)]-\frac{\pi^{2} m}{\eta} e^{-\eta|u|} \\
& +\ln \frac{|u|}{|u|+m^{-1}}+\tilde{C}_{2}(u)
\end{aligned}
$$

where

$$
\begin{aligned}
\tilde{C}_{2}(u)= & -\int_{0}^{1} d t \frac{\cos t-1}{t+m|u|}-\int_{1}^{\infty} d t \frac{\cos t}{t+m|u|} \\
& -\int_{0}^{\infty} d k \cos k u\left(\frac { 1 } { k ^ { 2 } } \left(\sqrt{k^{2}+4 \pi^{2} \omega_{k}^{2} e^{-2 x_{0}^{2} k^{2}}}\right.\right. \\
& \left.-2 \pi m)-\frac{1}{k+m}\right) .
\end{aligned}
$$

Inserting the expressions (A2) and (A5) in (39) and taking the limit $\eta \rightarrow 0$, we obtain

$$
\begin{aligned}
\left\langle y^{\prime} \mid y\right\rangle= & \frac{4 y y^{\prime}}{\left(2|y|+m^{-1}\right)\left(2\left|y^{\prime}\right|+m^{-1}\right)} \exp \left[2 \pi^{2} m\left(|y|+\left|y^{\prime}\right|-\left|y+y^{\prime}\right|-\left|y-y^{\prime}\right|\right)\right] \\
& \times \exp \left\{\tilde{C}_{2}(2 y)+\tilde{C}_{2}\left(2 y^{\prime}\right)+2\left[\tilde{C}_{1}\left(y+y^{\prime}\right)+\tilde{C}_{1}\left(y-y^{\prime}\right)-\tilde{C}_{1}(0)\right]\right\}
\end{aligned}
$$

It can be verified that the functions $\tilde{C}_{1}$ and $\tilde{C}_{2}$ are well behaved and vanish for large values of their argument. Once these functions are obtained by performing numerically the Fourier transforms (A3) and (A6), the baryon density of the two-body bound state in the soliton model can be computed by substituting (38), (A7), (6), and (9) in (13) and $(10)$.

[1] F. J. Ynduráin, Quantum Chromodynamics (SpringerVerlag, New York, 1983).

[2] P. J. Mulders, Phys. Rep. 185, 83 (1990).

[3] F. E. Close, An Introduction to Quarks and Partons (Academic Press, London, 1979).

[4] M. Betz, G. Krein, and Th. A. J. Maris, Nucl. Phys. A437, 509 (1985); G. Krein and Th. A. J. Maris, Phys. Rev. C 36, 365 (1987); P. Hoodbhoy and R. L. Jaffe, Phys. Rev. D 35, 113 (1987); P. Hoodbhoy, Nucl. Phys. A465, 637 (1987); T. de Forest and P. J. Mulders, Phys. Rev. D 35, 2849 (1987).

[5] S. G. Rajeev, Phys. Rev. D 29, 2944 (1984); I. Zahed and G. E. Brown, Z. Phys. A 335, 349 (1990).

[6] T. H. R. Skyrme, Proc. R. Soc. London 247, 260 (1958); 260, 127 (1961); 262, 237 (1961).

[7] G. 't Hooft, Nucl. Phys. B75, 461 (1974).
[8] E. Witten, Nucl. Phys. B160, 57 (1979).

[9] I. Zahed and G. E. Brown, Phys. Rep. 142, 1 (1986); V. G. Makhan'kov, Yu. P. Rybakov, and V. I. Sanyuk, Usp. Fiz. Nauk 162, 1 (1992) [Sov. Phys. Usp. 35, 55 (1992)].

[10] S. Mandelstam, Phys. Rev. D 11, 3026 (1975).

[11] P. Goddard and P. Mansfield, Rep. Prog. Phys. 49, 725 (1986).

[12] D. Hadjimichef, M.Sc. dissertation, Universidade Federal do Rio Grande do Sul, Porto Alegre, Brazil, 1991; D. Hadjimichef and M. Betz, in Current Topics in Nuclear Physics and Quantum Field Theory: Festschrift for Th. A. J. Maris, edited by D. Dillenburg, G. Jacob, P. Kitching, and C. A. Z. Vasconcellos (Universidade Federal do Rio Grande do Sul, Porto Alegre, Brazil, 1994).

[13] J. R. Klauder and B. S. Skagerstam, Coherent States (World Scientific, Singapore, 1987). 